\title{
Correction to: Confocal Microscopy
}

\section{Joseph Brzostowski and Haewon Sohn}

Correction to: Joseph Brzostowski and Haewon Sohn (eds.), Confocal Microscopy: Methods and Protocols, Methods in Molecular Biology, vol. 2304, https://doi.org/10.1007/978-1-0716-1402-0

Chapter 15 "Method for Acute Intravital Imaging of the Large Intestine in Live Mice" was previously published with incorrect ESM videos and figure captions. This has now been rectified in the revised version of this book. 